\title{
Function of smooth muscle cGMP-dependent protein kinase type I in a mouse model of restenosis
}

\author{
Robert Lukowski*1, Pascal Weinmeister ${ }^{1}$, Annette Vogl ${ }^{1}$, Susanne Feil ${ }^{1}$, \\ Michael Gotthardt ${ }^{2}$, Joachim Herz ${ }^{3}$, Steffen Massberg ${ }^{4}$, Franz Hofmann ${ }^{1}$ and \\ Robert Feil ${ }^{1}$
}

\author{
Address: ${ }^{1}$ Institut für Pharmakologie und Toxikologie, TU München, Germany, ${ }^{2}$ Max-Delbrück-Centrum für Molekulare Medizin, Berlin-Buch, \\ Germany, ${ }^{3}$ Department of Molecular Genetics, UT Southwestern, Dallas, USA and ${ }^{4}$ Deutsches Herzzentrum, TU München, Germany \\ Email: Robert Lukowski* - Lukowski@ipt.med.tu-muenchen.de \\ * Corresponding author
}

from 2nd International Conference of cGMP Generators, Effectors and Therapeutic Implications Potsdam, Germany, 10-12 June, 2005

Published: 16 June 2005

BMC Pharmacology 2005, 5(Suppl I):P32 doi:I0.II86/I47I-22I0-5-SI-P32

During vascular proliferative diseases, such as atherosclerosis and restenosis, smooth muscle cells (SMCs) from the vessel wall undergo phenotypic changes and show increased growth potential. The signaling molecule nitric oxide (NO) exerts both beneficial and deleterious effects on vessel remodeling, but the molecular pathways of vascular NO signaling are not well understood. The analysis of mouse mutants indicated that the NO/cGMP/cGMPdependent protein kinase type I (cGKI) pathway accelerates SMC growth in atherosclerotic plaques in vivo and in primary aortic SMCs in vitro. These results oppose the common view that cGKI signaling mediates vasculo-protective effects. To resolve this controversy, we used the Cre/lox system to generate SMC-specific cGKI knockout mice and performed unilateral carotid ligation in these animals. It has been shown previously that NO signaling plays a key role in this model of restenosis after mechanical vessel injury. Surprisingly, we could not detect any differences, based on the morphometric analysis of the neointima/media ratio, in the vascular response to injury between SMC-specific cGKI mutants and control mice at different time points after injury. Thus, smooth muscle cGKI is apparently not essential for vessel remodeling in this particular model of restenosis. Together, the analysis of cGKI-deficient mouse models of atherosclerosis and restenosis suggests that the role of smooth muscle cGKI in vascular remodeling might be context-specific, being more important in atherosclerosis than in restenosis. 